\title{
LA FORMACIÓN DEL PROFESORADO COMO DOCENTE EN LOS ESPACIOS VIRTUALES DE APRENDIZAJE
}

\author{
Begoña Gros Salvat \\ Universidad de Barcelona, España \\ Juan Silva Quiroz \\ Universidad de Santiago de Chile, Chile
}

\section{INTRODUCCIÓN}

Como señala Martínez (2003), la educación ha podido mantener con pocas variaciones su modelo clásico de enseñanza. De esta forma ha sobrevivido a los avances de la sociedad. Sin embargo las tecnologías de la información y la comunicación (TIC), plantean nuevos escenarios, que requieren una revisión profunda de la educación en sus diversos aspectos. En efecto la modalidad de enseñanza, las metodologías, la forma de acceder y adquirir conocimientos, los recursos utilizados, entre otras aspectos son afectadas por estas tecnologías.

La inserción de las TIC en los contextos educativos pueden reportar beneficios para el sistema educativo en su conjunto, alumnos, docentes y la comunidad educativa en general. En el caso de los docentes, las tecnologías ponen a su disposición diversos recursos electrónicos: software, documentos, página web, etc, facilitan la participación en redes de docentes, apoyan el trabajo de proyectos en forma colaborativa con otros centros educativos (Harasim et.al., 2000, Hepp, 2003; Crook, 1998). Una de las posibilidades emergentes derivadas de estas tecnologías instaladas en los centros educativos, es el uso de entornos virtual de aprendizaje (EVA) para apoyar la labor docente, extendiendo la clase más allá de las fronteras del aula. Los entornos virtuales de aprendizaje son además útiles para que los docentes puedan formarse de manera continua, participando de experiencias de formación centradas en perspectivas educativas constructivista de raíces socio culturales, donde la interacción con los pares, la reflexión y el construir conocimiento en forma colaborativa son aspectos centrales.

La formación inicial del profesorado no debería ignorar estos nuevos espacios de aprendizaje. La actuación del profesorado no puede pensarse sólo en un aula situada en un espacio físico, entre los muros del aula. Por ello, el rol del profesorado va a ir cambiando notablemente, lo que supone una formación mucho más centrada en el diseño de las situaciones y contextos de aprendizaje, en la mediación y tutorización, y en las estrategias comunicativas.

El dbjetivo fundamental de este artículo es precisamente analizar las necesidades formativas del profesorado en su formación inicial y continua teniendo como eje central la creación de espacios virtuales de aprendizaje.

\section{LA FORMACIÓN DEL PROFESORADO COMO DISEÑADOR DE NUEVOS ENTORNOS PARA EL APRENDIZAJE}

Papert (1996) para ejemplificar la lentitud de los cambios en las instituciones educativos establece la siguiente comparación. Imaginemos que hace un siglo hubiésemos congelado a un cirujano y a un maestro 
y ahora les volviéramos de nuevo a la vida. El cirujano entraría en la sala de operaciones y no reconocería ni el lugar ni los objetos y se sentiría totalmente incapacitado para actuar. ¿Qué pasaría con el maestro? Como señala Papert, el profesor reconocería el espacio como una clase y todavía encontraría una tiza y una pizarra con la cual empezar a enseñar.

Esta concepción de aula y de enseñanza es la que todavía está presente en la mayor parte de las escuelas. Sin embargo, urge cambiar este modelo formativo e incorporar en la formación del profesorado las herramientas para capacitarlo como un profesional que esté más cerca de ser un trabajador del conocimiento, un diseñador de entornos de aprendizaje, que un mero transmisor de información.

Los profesores que formamos se van a encontrar con alumnos que pertenecen a una nueva generación, una generación digital en la cual la información y el aprendizaje ya no esta relegada a los muros de la escuela ni es ofrecida por el profesor de forma exclusiva.

El principal problema de los profesores de la generación digital es que la sociedad actual ha cambiado de forma muy rápida y el profesorado se encuentra con una situación complicada: se han producido muy pocos cambios en cuanto a la estructura y la gestión de la escuela mientras que la sociedad ha cambiado de forma rápida, los niños actuales necesitan otro tipo de formación. Los profesores se han formado y se están formando con una cultura y una visión del significado de su profesión que ya ha cambiado.

El profesor dentro de la institución escolar tiene una cierta libertad para poder introducir mejoras o no modificar su práctica. No obstante, aunque puedan ver cómo la escuela tiene que cambiar, cómo necesitan mayor formación para afrontar los cambios carecen de capacidad de introducir modificaciones a la vez que la formación depende - en la mayoría de los casos- totalmente de su voluntad. Como señala C. Marcelo (2002), el aislamiento de los profesores está favorecido evidentemente por la arquitectura escolar, que organiza las escuelas en módulos estándar, así como por la distribución del tiempo y el espacio, y la existencia de normas de independencia y privacidad entre los profesores. "El aislamiento, como norma y cultura profesional tiene ciertas ventajas y algunos evidentes inconvenientes para los profesores ya que aunque facilita la creatividad individual y libera a los profesores de algunas de las dificultades asociadas con el trabajo compartido, también les priva de la estimulación del trabajo por los compañeros, y se deja de recibir el apoyo necesario para progresar a lo largo de la carrera" (Marcelo: 2002).

Los cambios que se están produciendo en la sociedad inciden en la demanda de una redefinición del trabajo del profesor y de la profesión docente, de su formación y de su desarrollo profesional. Los roles que tradicionalmente han asumido los docentes enseñando un currículum caracterizado por contenidos académicos hoy en día resultan inadecuados. A los alumnos les llega la información por múltiples vías ( la televisión, radio, Internet, etc.) y los profesores no pueden ignorar esta realidad. Salomon ofrece su metáfora respecto a esta modificación del rol del profesor desde transmisor de información, el solista de una flauta al frente de una audiencia poco respetuosa, al de un diseñador, un guía turístico, un director de orquesta (1992:42). Bajo esta perspectiva, el papel del profesor debería de cambiar desde una concepción puramente distribuidora de información y conocimiento hacia una persona que es capaz de crear y orquestar ambientes de aprendizaje complejos, implicando a los alumnos en actividades apropiadas, de manera que los alumnos puedan construir su propia comprensión del material a estudiar, y acompañándolos en el proceso de aprendizaje. 
Comprender lo que se aprende y aprender a aprender son algunos de los aspectos más repetidos en toda la bibliografía sobre el tema, y ello es aplicable tanto a los estudiantes como a los propios profesores. Los cambios en los profesores no pueden hacerse al margen de cómo $æ$ comprende el aprendizaje de los propios profesores y, esta comprensión debe presidir los planes de formación inicial y continua del profesorado. Estos cambios se concretan en una forma diferente de entender el proceso educativo tal y como mostramos en la siguiente figura:

\begin{tabular}{|l|l|l|}
\hline & Enseñanza tradicional & Sociedad informacional \\
\hline Aprendizaje & $\begin{array}{l}\text { Transmisión de la información } \\
\text { Modelo jerárquico }\end{array}$ & $\begin{array}{l}\text { Centrado en el aprendiz } \\
\text { Situado } \\
\text { Cooperativo }\end{array}$ \\
\hline Modelo de enseñanza & $\begin{array}{l}\text { Transmisión y comprobación de } \\
\text { que la recepción sea igual a lo } \\
\text { transmitido }\end{array}$ & $\begin{array}{l}\text { Andamiaje } \\
\text { Evaluación } \\
\text { (transformación como valor) }\end{array}$ \\
\hline Currículum & Fijo & Flexible \\
\hline Tareas & $\begin{array}{l}\text { Materiales secuenciados } \\
\text { Fijas }\end{array}$ & Auténticas \\
\hline Agrupación & $\begin{array}{l}\text { Individual } \\
\text { Competitiva }\end{array}$ & $\begin{array}{l}\text { Colaboración } \\
\text { Comunidades de aprendices }\end{array}$ \\
\hline Herramientas & $\begin{array}{l}\text { Libros } \\
\text { Papel y lápiz }\end{array}$ & $\begin{array}{l}\text { Múltiples formatos: } \\
\text { multimedia, Internet, etc. }\end{array}$ \\
\hline
\end{tabular}

Es evidente que casi todos reconocemos los aspectos presentes en la columna referida a la enseñanza tradicional mientras que los elementos descritos en el ámbito de la sociedad informacional todavía son incipientes y la formación inicial del profesorado debe diseñarse de tal modo que permita crear las competencias adecuadas para formar profesionales que no sólo dominen la didáctica especifica de las diferentes materiales sino también una serie de elementos educativos transversales que todo profesional de la educación debe adquirir.

Junto a la capacidad de aprender, un elemento que se viene considerando también de gran importancia es la capacidad para dar respuesta a los desafíos actuales de las escuelas es la capacidad de liderazgo de los profesores. Se está pidiendo un profesor entendido como un "trabajador del conocimiento", diseñador de ambientes de aprendizaje, con capacidad para rentabilizar los diferentes espacios en donde se produce el conocimiento. Y una profesión docente caracterizada por lo que Wenger (2001) denomina una comunidad de práctica a través de la que la experiencia individual pueda convertirse en colectiva. Una profesión que necesita cambiar su cultura profesional, marcada por el aislamiento y las dificultades para aprender de otros y con otros; en la que está mal visto pedir ayuda o reconocer dificultades.

Empieza a haber experiencias interesantes en el que se muestra cómo el uso de las tecnologías puede facilitar enormemente el trabajo de los profesores y su propia formación. La creación de comunidades virtuales para compartir recursos y conocimientos así como la creación de espacios de práctica compartidos son una muestra ${ }^{1}$

\footnotetext{
1 Algunos profesores han conseguido familiarizarse a través de su implicación en la evaluación del software, logrando así convertirse en usuarios mejor informados, como en el sistema alemán/austríaco SODIS (Software Documentation and Information System). En la iniciativa del Reino Unido TEEM (Teachers Evaluating Educational Multimedia), los profesores informan sobre el uso del software en el aula con fines de evaluación.
} 
Tal y como se expone en el informe europeo "Learning to bridge the digital divide" (2000), las TIC permiten una nueva complementariedad entre el aprendizaje formal en la escuela y el aprendizaje informal fuera de ella. Para alcanzar esta formación es necesario que el profesorado sea formado utilizando las herramientas y metodologías que después deberá aplicar con sus estudiantes. En este sentido, un elemento clave para la formación es el aprendizaje de las estrategias para gestionar entornos virtuales de aprendizaje.

Como hemos señalado previamente, no se trata sólo de aprender entre los muros del aula sino de diseñar espacios virtuales que permitan el aprendizaje. Diseñar un entorno virtual y actuar como formador (tutor) no es tarea fácil y debería formar parte del aprendizaje inicial del profesorado.

\section{EL ROL DEL TUTOR EN LOS ENTORNOS VIRTUALES DE APRENDIZAJE}

El impacto de las TIC en la comunicación es tal que se denomina Comunicación Mediada por Computador ( $\mathrm{CMC})$ a todas estas instancias de comunicación sincronas o asincronas realizadas por medios de recursos tecnológicos. En los últimos años ha crecido rápidamente el uso de $\mathrm{CMC}$, especialmente en su modalidad asíncrona (Tolmie y Boyle, 2000). Esta herramienta comunicativa incorporada de modo apropiado en los procesos de enseñanza o formación, y bien asistidas pueden favorecer la colaboración "un proceso interactivo y colectivo de producción de conocimiento en donde los alumnos producen el conocimiento activamente formulando las ideas por escrito que son compartidas y construidas a partir de las reacciones y respuestas de los demás" (Harasim et.al. 2000, p.24). De esta forma nos encontramos frente a la posibilidad de crear entornos de aprendizaje que posibilitando el trabajo colaborativo y potenciando la construcción de conocimiento en una comunidad de aprendizaje. Se promueve entonces espacios para la reflexión, accesible a toda hora, adaptables al ritmo de aprendizaje individual y por sobre todo opuestos a la clásica transmisión de conocimiento del profesor al alumno.

Las experiencias educativas que utilicen estos entornos virtuales de aprendizaje, tanto si se ubican en escenarios de enseñanza a distancia, presencial o mixta, requieren una redefinición de los elementos organizativos del aprendizaje, en relación a: los agentes involucrados (profesores, participantes, administrativos); los espacios donde se lleva a cabo las actividades formativos (casa, centro educativo, aulas informáticas, lugar de trabajo); los tiempos; y secuencias de aprendizajes (Pérez, 2002). En relación al rol del tutor se plantean nuevas compet encias y habilidades: deben estar preparado para generar un dialogo efectivo con los participantes y entre los participantes, de modo que se favorezca el aprendizaje activo, la construcción de conocimiento cooperativo y/o colaborativo.

Para el correcto funcionamiento de un espacio virtual que facilite la interacción social y la construcción de conocimiento, se requiere siempre la intervención de un profesor/tutor que realice el seguimiento y la moderación. Esta permite mantener "vivos" los espacios comunicativos, facilitar el acceso a los contenidos, animar el dialogo entre los participantes, ayudándoles a compartir su conocimiento y a construir conocimiento nuevo. Estos profesionales son fundamentales en le éxito de las experiencias de enseñanza y formación que utilizan CMC para la colaboración (Cabero, 2001; Salmon, 2000; Ryan et.al., 2000, Swan et.al 2000). Harasim y otros (2000), señalan que en la educación y formación tradicional el profesor dirige la instrucción, hace las preguntas y marca el ritmo de la clase, en cambio el aprendizaje en grupo en red está centrado en el alumno y requiere un papel diferente del profesor, más cercano al 
ayudante que al encargado de impartir lecciones. "el énfasis tiene que estar en el propio proceso intelectual del alumno y en el aprendizaje en colaboración" (Harasim y otros, 2000, p. 198)

\subsection{El rol moderador del tutor en los espacios virtuales de aprendizaje}

Para Paulsen y Mason, los roles fundamentales del moderador se pueden clasificar dentro de lo organizativo, social e intelectual. Lo organizativo supone preparar la conferencia y estimular la participación, requerir la participación regular en el proceso, invitar a expertos a que puntualmente se incorporen al proceso, u ocasionalmente de hacer que los estudiantes conduzcan la discusión, establecer la agenda de la conferencia, determinar los objetivos de la discusión, el itinerario y la especificación de las reglas que la marcarán. En el aspecto social crear un ambiente amistoso y socialmente positivo que sea propicio para el desarrollo de un ambiente de aprendizaje positivo en comunidad. En el aspecto intelectual enfocar los puntos fundamentales, recapitular y evaluar las intervenciones (Paulsen, 1995; Mason, 1991, en Cabero 2001)

De acuerdo a Ryan y otros (2000) varios autores concuerdan en caracterizar los roles y responsabilidades del moderador en la conferencia computacional en cuatro categorías: pedagógica, social, administrativa y técnica. En lo pedagógica, el tutor es un facilitador que contribuye con conocimiento especializado, focaliza la discusión en los puntos críticos, hacer las preguntas y responder a las contribuciones de los participantes, le da coherencia a la discusión, sintetiza los puntos destacando los temas emergentes. El lo social necesita habilidades para crear una atmósfera de colaboración que permita generar una comunidad de aprendizaje. En el aspecto técnico debe garantizar que los participantes se sienten cómodos con el software y si es necesario apoyarlos. En cuanto a lo administración conocer e software para poder generar subconferencias, grupos de trabajo, y mover o borrar mensajes de la conferencia.

Uno de los principales roles del tutor es el de "moderador", de la discusión. Barbera y otros (2001) ha sintetizado las tareas del moderador en e desarrollo de la discusión en tres etapas: planificación, intervención en el desarrollo, y cierre. Estas tres etapas y las tareas asociadas da cada una de ellas se presenta en el siguiente esquema.

\begin{tabular}{|l|l|}
\multicolumn{1}{|c}{ Planificación de la } \\
discusión
\end{tabular}

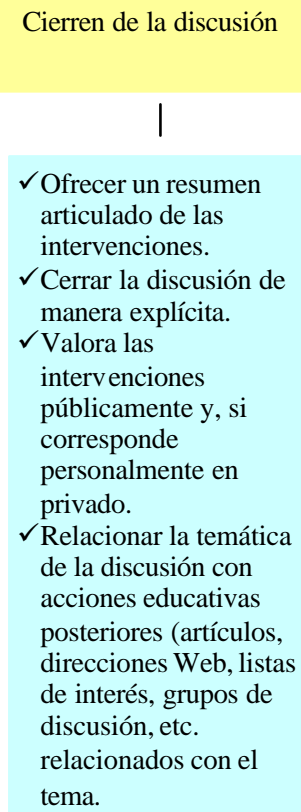


En la primera etapa el moderador prepara la discusión y los elementos que pueden ayudar a moderarla, así como las indicaciones para organizar y facilitar la intervención de los participantes. En la segunda etapa, se articula la discusión y se produce el intercambio y construcción de conocimiento, el rol del moderador es facilitar esta construcción, cautelar que la discusión tome un rumbo de acuerdo a los propósitos diseñados, entregar retroalimentación. La tercera etapa cierra la discusión, resumiéndose los principales aportes, es una toma de conciencia del proceso y la construcción realizada, cómo esta toma sentido en la construcción personal y su proyección con tareas educativas posteriores.

Salmon(2000) ha contribuido en gran medida a comprender el rol del moderador y sus cualidades y habilidades. En efecto basándose en una investigación acción la profesora Gilly Salmon de la Open University, ha establecido un modelo para la moderación de CMC.
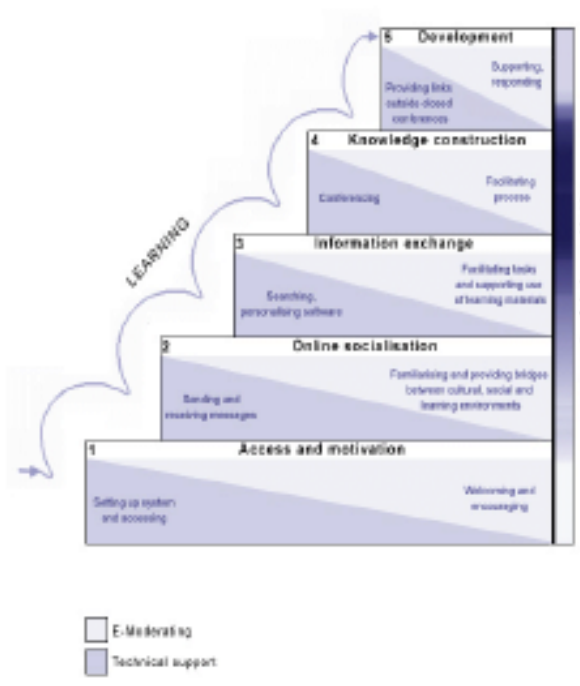

El modelo se presenta en forma de una escala en la cual aparecen por cada nivel dos tipos de habilidades, la moderación en el ambiente virtual (E-Moderating) y el soporte técnico. También se muestra en la barra vertical de la derecha el grado de interactividad durante el desarrollo de las etapas que parte siendo muy poco en la etapa de acceso y motivación -se comunican con uno o dos y pocos mensajes-, aumenta lentamente en la etapa de socialización - se comunican más entre ellos y con mayor frecuencia- y se intensifica en las etapas de intercambio de información y construcción de conocimiento - es donde participa un mayor número y con más intensidad-, volviendo a decrecer en la etapa de desarrollo, esto último porque esta es de carácter más personal, produciéndose menos comunicación entre ellos. Estas etapas ilustran la interacción entre competencia y factores afectivos como crecimiento de la confidencia, motivación y dinámica de grupo (Macdonald, 2003).

Salmon, incorpora el concepto de E-Moderator para referirse a un tutor especializado en la moderación y el E-moderating, para referirse al proceso de moderación de la conferencia en el entorno virtual.

- Acceso y Motivación (Etapa 1): En esta etapa tanto para el moderador como para los participantes es esencial acceder al ambiente y poder adquirir la habilidad para utilizar CMC, estos aspectos son requisitos previos esenciales para la participación en la conferencia. El primer problema al que se enfrentan los participantes se relaciona con el acceso a la red y el espacio de la conferencia. Muchos necesitan ayuda que les permita solucionar los problemas 
técnicos con el hardware, software, acceso a la red y en algunas ocasiones con las claves de acceso, de allí la importancia de una ayuda en línea o telefónica. El moderador debe dar este soporte que es crucial para los participantes.

- Socialización (Etapa 2): En esta etapa es en la cual los participantes establecen sus identidades en línea y comienzan a interactuar. La tecnología crea o facilita las instancias para la socialización, pero esta no ocurrirá sino existe una intervención sensible y apropiadas por parte del E-moderador. En esta etapa los participantes se habitúan a usar la CMC para comunicarse con los pares estableciendo una comunidad de aprendizaje que les permitan sentirse trabajando juntos en tareas comunes.

- Compartir información (Etapa 3): En esta etapa comienza a producirse el intercambio de información, las interacción crece producto de que hay más participantes que hacen aportes y con mayor frecuencia, se aporta información relevante para los otros. La información crece y se comienza a percibir un cierto desorden y de saturación por tanta información. Los participantes desarrollan diversas estrategias para afrontar la sobrecarga de información y el tiempo que implica manejarla: no intentan leer todo, leer lo que les interesa, intentar leer todo e invierten cuando les parece oportuno, leen todo pero rara vez responden. E-moderador necesita mirar $\mathrm{c} / \mathrm{u}$ de estas estrategias, ofreciendo ayuda y direccionamiento a los participantes. Los participantes que logran organizarse y aprender rápidamente a compartir el volumen de trabajo en equipo, afrontan mejor esta problemática.

- Construcción de conocimiento (Etapa 4). En la etapa cuatro, el grupo se relaciona a través de la discusión, no se comunica para recibir información o entregar información (como en la etapa 3), sino que se vuelve activo en la construcción de conocimiento. En esta etapa los participantes comienzan a interactuar con los otros en forma más participativa, formulando y escribiendo sus ideas o comprensión de los contenidos. Se produce una mayor interacción porque ellos leen los mensajes de los otros y responden, produciéndose frecuentemente un dialogo fructífero, muchos participantes logran generar aprendizajes activos, extendiendo sus puntos de vistas y apreciarlos desde diferentes perspectivas.

- Desorrollo (Etapa 5) En la etapa cinco, los participantes buscan más ventajas del sistema para ayudarles a alcanzar metas personales, exploran cómo integrar CMC en otras formas de aprender y su reflejo en los procesos de aprendizaje. Se vuelven más críticos con el ambiente tecnológico que soporta la CMC y su funcionamiento, así como el actuar del tutor, lo que puede dificulta en algunos casos la negociación. Es una etapa de construcción individual del conocimiento, que permite a los participantes explorar sus propios pensamientos y procesos de construcción de conocimiento, a partir no sólo de los tópicos del área de estudio, sino también y especialmente de las interacciones, con el moderador y los pares.

\subsection{Cualidades y habilidades de tutor}

Los moderadores necesitan contar con una serie de habilidades y cualidades que les permitan cumplir su rol. "Se necesitan unas habilidades especiales para preparar y presentar un programa de estudios interactivo y participativo de verdad, así como para facilitar y gestionar la participación" (Moore, 
2001). Estas se presentan en los cuatro ámbitos antes descritos: pedagógico, social, técnico y administrativo.

En lo pedagógico el tutor acompaña, media y retroalimenta al estudiante en su proceso de formación, conduce el aprendizaje individual y grupal, orientando y aconsejando cuando el alumno o el grupo lo necesite. El lo social, debe poseer habilidades sociales que le permitan crear y mantener una comunidad de aprendizaje donde se respire una atmósfera agradabe, debe ser acogedor, empático y estar siempre dispuesta a ayudar. En lo técnico, debe posees habilidades mínimas de carácter general relacionadas con el uso de la tecnología, los computadores y las redes. Además necesitará habilidades técnicas para intervenir en el sistema de conferencia. En lo administrativo utilizar las herramientas que provea el ambiente para este propósito y que permitan crear y gestionar la conferencia, hacer un seguimiento a la participación individual de alumno y general del grupo, y administrar los equipos de trabajo.

En relación a la moderación Salmon (2001), resumen las características y habilidades del Emoderador en el siguiente cuadro. Este requiere características y habilidades personales las cuales asociadas a ciertas cualidades personales permiten al tutor estar preparado para cumplir adecuadamente el rol moderador.

\begin{tabular}{|c|c|c|c|c|c|c|}
\hline $\begin{array}{l}\text { Cualidad/ } \\
\text { Característica }\end{array}$ & Seguro & Constructivo & De desarrollo & Facilitador & $\begin{array}{l}\text { Compartir } \\
\text { conocimiento }\end{array}$ & Creativo \\
\hline $\begin{array}{l}\text { Comprensió } \\
\mathrm{n} \text { de los } \\
\text { procesos } \\
\text { online }\end{array}$ & $\begin{array}{l}\text { Seguro en } \\
\text { proveer un } \\
\text { enfoque para } \\
\text { la } \\
\text { conferencia, } \\
\text { juzgar el } \\
\text { interés de los } \\
\text { participantes, } \\
\text { experimentar } \\
\text { con } \\
\text { diferentes } \\
\text { acercamiento } \\
\text { s, y siendo un } \\
\text { modelo del } \\
\text { papel. }\end{array}$ & $\begin{array}{l}\text { Capaz para } \\
\text { construir } \\
\text { confianza y } \\
\text { propósito en } \\
\text { línea, } \\
\text { Para saber } \\
\text { quién debe } \\
\text { ser en línea y } \\
\text { lo que ellos } \\
\text { deben estar } \\
\text { haciendo }\end{array}$ & $\begin{array}{l}\text { Habilidad para } \\
\text { desarrollar y } \\
\text { activar a otros, } \\
\text { actuar como } \\
\text { catalizador, } \\
\text { sostener la } \\
\text { discusión, } \\
\text { resumir, } \\
\text { reiterar el } \\
\text { desafío, } \\
\text { monitor en la } \\
\text { comprensión y } \\
\text { equivocación, } \\
\text { dar feedback }\end{array}$ & $\begin{array}{l}\text { Saber cuándo } \\
\text { controlar los } \\
\text { grupos, } \\
\text { cuándo } \\
\text { permitirles ir, } \\
\text { cómo llevarse } \\
\text { con los no } \\
\text { participantes, } \\
\text { saber cómo } \\
\text { marcha la } \\
\text { discusión y } \\
\text { usar del } \\
\text { tiempo en } \\
\text { línea }\end{array}$ & $\begin{array}{l}\text { Capacidad para } \\
\text { explorar ideas, } \\
\text { desarrollar } \\
\text { argumentos, } \\
\text { promover los } \\
\text { líneas valiosos, } \\
\text { cerrar las líneas } \\
\text { improductivos, } \\
\text { escoja cuándo } \\
\text { archivar, } \\
\text { construya una } \\
\text { comunidad de } \\
\text { aprendizaje }\end{array}$ & $\begin{array}{l}\text { Capacidad } \\
\text { para usar una } \\
\text { gama de } \\
\text { CMC, desde } \\
\text { actividades } \\
\text { estructuradas } \\
\text { a libres } \\
\text { generación } \\
\text { de } \\
\text { discusiones, y } \\
\text { a evaluar y } \\
\text { juzgar el éxito } \\
\text { de la } \\
\text { conferencia. }\end{array}$ \\
\hline $\begin{array}{l}\text { Habilidades } \\
\text { técnicas }\end{array}$ & $\begin{array}{l}\text { Comprender } \\
\text { a nivel de } \\
\text { usuario el uso } \\
\text { del software, } \\
\text { razonable } \\
\text { habilidades } \\
\text { en el uso de } \\
\text { las TIC, buen } \\
\text { acceso. }\end{array}$ & $\begin{array}{l}\text { Capaz para } \\
\text { apreciar las } \\
\text { básicas } \\
\text { estructuras } \\
\text { de CMC, y la } \\
\text { WWW y el } \\
\text { potencial de } \\
\text { Internet para } \\
\text { el } \\
\text { aprendizaje }\end{array}$ & $\begin{array}{l}\text { Conocimiento } \\
\text { cómo a usar } \\
\text { las } \\
\text { características } \\
\text { especiales de } \\
\text { software para } \\
\text { e-moderador } \\
\text { ej, control, } \\
\text { archivos. }\end{array}$ & $\begin{array}{l}\text { Capacidad } \\
\text { para usar las } \\
\text { característica } \\
\text { s del software } \\
\text { para explorar } \\
\text { el uso de los } \\
\text { aprendices ej: } \\
\text { historia de } \\
\text { mensajes }\end{array}$ & $\begin{array}{l}\text { Capacidad para } \\
\text { lazos entre CMC } \\
\text { y otros } \\
\text { elementos del } \\
\text { programa de } \\
\text { aprendizaje }\end{array}$ & $\begin{array}{l}\text { Capacidad } \\
\text { para utilizar } \\
\text { las facilidades } \\
\text { del software } \\
\text { para creary } \\
\text { manipular } \\
\text { conferencias } \\
\text { y para } \\
\text { generar un } \\
\text { ambiente de } \\
\text { aprendizaje } \\
\text { online. }\end{array}$ \\
\hline $\begin{array}{l}\text { Habilidades } \\
\text { comunicativa } \\
\text { s on line }\end{array}$ & $\begin{array}{l}\text { Seguro de } \\
\text { ser cortez, } \\
\text { educado, y } \\
\text { respetuoso al } \\
\text { escribir las } \\
\text { comunicacion } \\
\text { es on line }\end{array}$ & $\begin{array}{l}\text { Capaz para } \\
\text { escribir } \\
\text { concisos, } \\
\text { enérgicos y } \\
\text { atractivos } \\
\text { mensajes } \\
\text { online }\end{array}$ & $\begin{array}{l}\text { Capacidad } \\
\text { para } \\
\text { comprometers } \\
\text { e en el trabajo } \\
\text { online con la } \\
\text { gente (no la } \\
\text { máquina o el } \\
\text { software) }\end{array}$ & $\begin{array}{l}\text { Capacidad } \\
\text { para } \\
\text { interactuar a } \\
\text { través del e- } \\
\text { mail y } \\
\text { conferencia y } \\
\text { lograr la } \\
\text { interacción } \\
\text { entre los } \\
\text { otros. }\end{array}$ & $\begin{array}{l}\text { Capacidad para } \\
\text { la diversidad con } \\
\text { sensibilidad } \\
\text { cultural }\end{array}$ & $\begin{array}{l}\text { Capacidad } \\
\text { para } \\
\text { comunicarse } \\
\text { agradableme } \\
\text { nte sin la } \\
\text { señal visual }\end{array}$ \\
\hline
\end{tabular}




\begin{tabular}{|c|c|c|c|c|c|c|}
\hline $\begin{array}{l}\text { Contenido } \\
\text { experto }\end{array}$ & $\begin{array}{l}\text { Seguridad de } \\
\text { poseer } \\
\text { conocimiento, } \\
\text { y experiencia } \\
\text { para } \\
\text { compartir, y } \\
\text { dispuesto y } \\
\text { capaz a } \\
\text { agregar } \\
\text { contribucione } \\
\text { s propias }\end{array}$ & $\begin{array}{l}\text { Capaz de } \\
\text { animar las } \\
\text { legitimas } \\
\text { contribucione } \\
\text { s de otros. }\end{array}$ & $\begin{array}{l}\text { Capacidad } \\
\text { para activar } \\
\text { debates } \\
\text { proponiendo y } \\
\text { cuestionando. }\end{array}$ & $\begin{array}{l}\text { Tener } \\
\text { autoridad } \\
\text { para otorgar } \\
\text { normas a los } \\
\text { estudiantes } \\
\text { para sus } \\
\text { participacione } \\
\text { s y } \\
\text { contribucione } \\
\text { s en CMC. }\end{array}$ & $\begin{array}{l}\text { Conocer acerca } \\
\text { de la } \\
\text { disponibilidad de } \\
\text { recursos (ej. en } \\
\text { la www) y enviar } \\
\text { a los } \\
\text { participantes a } \\
\text { ellos. }\end{array}$ & $\begin{array}{l}\text { Capacidad } \\
\text { para avivar } \\
\text { conferencias } \\
\text { a través del } \\
\text { uso de } \\
\text { multimedia y } \\
\text { recursos } \\
\text { electrónicos. }\end{array}$ \\
\hline $\begin{array}{l}\text { Característic } \\
\text { as } \\
\text { personales }\end{array}$ & $\begin{array}{l}\text { Seguro en } \\
\text { ser decidido y } \\
\text { motivador } \\
\text { como e- } \\
\text { moderador }\end{array}$ & $\begin{array}{l}\text { Capaz de } \\
\text { establecer } \\
\text { una identidad } \\
\text { online como } \\
\text { e-moderador }\end{array}$ & $\begin{array}{l}\text { Capacidad } \\
\text { para } \\
\text { adaptarse a } \\
\text { nuevos } \\
\text { contextos de } \\
\text { enseñanza, } \\
\text { métodos, } \\
\text { audiencias y } \\
\text { roles. }\end{array}$ & $\begin{array}{l}\text { Mostrar } \\
\text { sensibilidad } \\
\text { para } \\
\text { relacionarse y } \\
\text { comunicarse } \\
\text { online }\end{array}$ & $\begin{array}{l}\text { Mostrar una } \\
\text { positiva actitud, } \\
\text { compromiso y } \\
\text { entusiasmo para } \\
\text { el aprendizaje } \\
\text { online. }\end{array}$ & $\begin{array}{l}\text { Sepa crear } \\
\text { una } \\
\text { comunidad de } \\
\text { aprendizaje } \\
\text { en línea útil, } \\
\text { pertinente }\end{array}$ \\
\hline
\end{tabular}

\subsection{Formación de los tutores}

Las habilidades que posee un docente en un ámbito presencial aún siendo un excelente docente no garantizan el éxito cuando se actúa entornos virtuales "no necesariamente un buen profesor en un entorno presencial, podrá tener buenos resultado como tutor en un ambiente virtual" (Moore 2001). Las habilidades exitosas de un profesor en entornos presenciales son insuficientes en los entornos virtuales (Salmon, 2000). Especialmente porque en la formación online se pierde los recursos gestuales que utiliza el profesor, siendo la comunicación escrita la que permite la interacción con los estudiantes.

Es necesario una formación del tutor para dotarlo de las habilidades necesarias para cumplir un adecuado rol en la moderación de la conferencia. Esta debería proporcionarle las habilidades necesarias para desenvolverse adecuadamente en los aspectos sociales, pedagógicos, técnicos y administrativos. Especialmente los pedagógicos y sociales. Un moderador que desee realizar bien su trabajo y quedar satisfecho por su actuación en particular y el logro de aprendizajes de sus estudiantes, debe tomar conciencia de lo relevante que es prepararse para cumplir eficientemente este rol, valorando sus particularidades, complejidades y la diferencia con los roles en otras formas de enseñanza y formación.

La formación en los aspectos técnicos y administrativos puede lograrse fácilmente en una par de talleres presenciales, o en un curso en la modalidad a distancia -tiene la particularidad que permite vivenciar el trabajo desde el rol del estudiante- o una combinación de estas dos. Sin embargo el aspecto más complicado son los aspectos pedagógicos y sociales, esto es a más largo plazo y es una mezcla entre capacitación y experiencia. Harasim propone un modelo en el cual los futuros moderadores trabajan en conjunto con moderadores experimentados, asumiendo paulatinamente responsabilidades partiendo por aquellas más básicas relacionadas con el soporte y las ayudas administrativas, para ir avanzando a las más complejas de animación de las discusiones (Harasim y otros, 2000).

Un aspecto a veces postergado a un segundo plano es la formación de tutores en los contenidos que se trabajan en el ambiente virtual. Especialmente cuando el tutor no ha participado en la generación del curso, aspecto habitual en las prácticas de formación a distancia, en las cuales esta tarea la desarrolla un equipo pedagógico que luego no interviene en el trabajo de los estudiantes. Si bien en un comienza las preguntas se relacionan con el ambiente virtual, aspectos administrativos asociados al mismo, ya entrada 
en materia se necesita conocimiento de los contenidos para poder guiar la discusión y asistir en la construcción del conocimiento. Luego es absolutamente necesario conocer por parte de los tutores los contenidos, las actividades propuestas, el sentido de los espacios de discusión, la finalidad de los trabajos, las estrategias metodológicas para su enseñanza en un entorno virtual que apuesta por una construcción y no transmisión de conocimientos. Silva y Oteiza (2002) reportan una experiencia de formación de tutores para un curso a distancia destinado a docentes de matemática de nivel secundario, donde ésta se realizó en la modalidad a distancia, utilizando la misma pataforma y ambientes de trabajo en la cual ellos actuarían posteriormente como tutores. Se configuró con los tutores una comunidad de aprendizaje inserta en un campus virtual, el programa de formación de tutores contemplo: introducción a la formación a distancia; una unidad del curso en el cual ellos actuarían como tutores; evaluación del aprendizaje en entornos virtuales.

Salmon(2001) propone para los tutores (E-moderadores) un modelo de capacitación de 5 etapas siguiendo el modelo de E-moderación descrito anteriormente, las etapas son: bienvenida, inducción, enseñanza, construcción de conocimiento y desarrollo. Cada etapa finaliza con una conferencia para discutir sus impresiones y avances en la etapa. Bienvenida tiene como propósito asegurarse que en su formación los aprendices puedan encontrarse con una conferencia, leyendo y enviando mensajes. Envían una pequeña descripción de ellos y conocen al resto de los participantes. Inducción permite al aprendiz conocer acerca de los protocolos y como relacionar a otros a través de este medio, adquiriendo las habilidades en las utilidades del software. Enseñanzaconcierne en dar y recibir información, en este sentido se entrega a los aprendices formación en la apertura de la conferencia y cómo "tejer" posterior los mensajes. Construcción de conocimiento se invita y estimula a los aprendices a discutir como ellos usarían CMC con sus propios estudiantes. Desarrollo se explora el uso de la Web en la enseñanza, construyendo confianza en los participantes y permitirles que consideren cómo podrían incorporar recursos Web en sus propias E-moderación. Este modelo puede usarse como andamiaje para la formación y desarrollo de Emoderadores (González y Salmon, 2002). La formación es complementada con un monitoreo de la actuación del aprendiz en la moderación de una conferencia real, por parte de pares o tutores que tienen completada con éxito la formación online.

Una estrategia señalada por varios autores (Harasim y otros 2000; Salmon 2000, entre otros) considera crear una comunidad de aprendizaje con los tutores, donde ellos compartan sus experiencias, los problemas encontrados, las estrategias utilizadas, etc. De esta forma estarán construyendo conocimiento en forma colaborativa para mejorar su trabajo y utilizando las mismas estrategias que deben desarrollar en sus estudiantes. Esta estrategia permite crear en los tutores un sentido de equipo, de pertinencia a la institución o programa que los acoge. También esta estrategia resulta útil en programas de formación masiva que contemplan replicas del mismo curso en forma simultanea y en el que intervienen un grupo de tutores, permitiéndoles crear una comunidad de tutores para compartir: inquietudes, problemas, estrategias implementadas con éxito, recibir apoyo del equipo creador de los contenidos, etc.

Existe un claro consenso que la actualización docente, es una de las claves para la implementación de procesos de enseñanza innovadores, que potencien más y mejores aprendizajes. Las TIC pueden apoyar los procesos de formación continua de los docentes a través de los espacios virtuales de aprendizaje. Creando instancias formativas donde la interacción, la colaboración y el aprendizaje en compañía de los pares y apoyados por un tutor, se transformen en ricos escenarios para la actualización docente. Estas instancias formativas pueden introducirse desde la formación inicial en las facultades de 
pedagogía hasta la formación permanente durante el desarrollo de la vida profesional. El que los docentes usen estos espacios virtuales para formarse, les permite conocer de forma más directa los roles que se esperan de un "profesor virtual", el que ellos podrían ejercer al incorporar esta modalidad de enseñanza en sus prácticas docentes.

Las experiencias de formación virtual de docentes, permite a estos profesionales acceder a actualizaciones en línea, diseñadas normalmente por equipos pedagógicos calificados y de cobertura a nivel nacional o internacional. Permiten al mismo tiempo la adquisición de parte de los profesores de nuevas competencias y habilidades para enfrentar en mejores condiciones una próxima experiencia en esta área. Este tipo de formación podría transformarse, en el futuro, en una de las principales fuentes de formación y actualización docente. Diversos países en distintos continentes están invirtiendo en esta modalidad. Sin embargo es necesario tener presente que las características específicas de la profesión docente, evitándose la utilización acrítica de modelos de otras industrias y profesiones (Grünberg , 2002). En este contexto el rol del tutor cobra renovado interés, pues el tutor es el "profesor virtual" de alumnos que se desempeñan como profesores en escenarios presenciales.

\section{CONCLUSIONES}

Las TIC están produciendo cambios en la formas de enseñanza y aprendizaje. En la forma en que los profesores y aprendices se relacionan con el conocimiento nuevo y las forma en que los agentes involucrados en el proceso educativo interactúan. Particularmente relevante resultan los efectos de los aspectos comunicativos y sus impactos en las modalidades de enseñanza a distancia y semipresencial, aunque también comienzan a tener efectos en las clases tradicional presencial. Estos espacios comunicativos ofrecen posibilidades de crear entornos de aprendizaje que permiten implementar estrategias de enseñanza y aprendizajes, en las cuales la interacción, posibilitan el trabajo colaborativo y la construcción de conocimiento en una comunidad de aprendizaje.

Son variados los potenciales pedagógicos del uso de los aspectos comunicativos de las TIC y también los factores que influyen en su éxito. Es necesario tener en cuenta estos elementos a la hora de incorporar esta herramienta en el diseño instructivo de un entorno virtual, pues la sola incorporación de estos espacios no garantiza la interacción, la colaboración ni la construcción de conocimiento. En este sentido al menos debe considerarse tres aspectos: el diseño de los espacios en la plataforma, la moderación del tutor y la relevancia de los temas a debatir.

La mayoría de las iniciativas innovadoras han sido realizadas de forma muy solitaria por profesores y profesoras entusiastas que dedicando mucho tiempo y esfuerzo han logrado introducir modificaciones metodológicas y tecnológicas. Esta labor no siempre es contagiosa y la actividad solitaria cel innovador acaba cansando. En este sentido, las innovaciones, la apropiación de la tecnología no puede ser responsabilidad de unos pocos profesores. Debe haber un mayor liderazgo por parte de los equipos de centro, la inclusión de la tecnología no puede ser algo que dependa de la voluntad del profesorado debería ser un objetivo a alcanzar por las escuelas como colectivo.

La generación de recursos tecnológicos, de contenidos educativos en red, la difusión de prácticas innovadoras es necesaria. Un modelo docente centrado en el estudiante requiere, por parte del profesor, de una mayor variedad de materiales y, por tanto, debe trabajar en red y en la red. Es necesario una formación 
del tutor para dotarlo de las habilidades necesarias para cumplir un adecuado rol en la moderación de la conferencia. Esta debería proporcionarle las habilidades necesarias para desenvolverse adecuadamente en los aspectos pedagógicos y sociales. Un moderador que desee realizar bien su trabajo y quedar satisfecho por su actuación en particular y el logro de aprendizajes de sus estudiantes, debe tomar conciencia de lo relevante que es prepararse para cumplir eficientemente este rol, valorando sus particularidades, complejidades y la diferencia con los roles en otras formas de enseñanza y formación.

Las redes tecnológicas sólo tienen sentido dentro de las redes sociales, si la escuela sigue siendo un elemento aislado nunca podrá incorporar la tecnología, es incompatible. El desarrollo de las redes supone un proceso evolutivo en el que la innovación y el aprendizaje es un aspecto central. El reto actual es que no sólo tienen que aprender los estudiantes sino las propias escuelas.

Consideramos fundamental que la formación inicial y continua del profesorado incorpore en sus planes y acciones, la capacitación requerida para el diseño y tutorización de los entornos virtuales de aprendizaje.

\section{REFERENCIAS}

BARBERÀ, E. (Coord), BADIA, A \& MOMINO, J.M. (2001). La incógnita de la educación a distancia, Barcelona:ICE-Horsori

BATES, A.W. (1995). Technology open learning and distance education, London/NewYork: Routledge.

CABERO, J. (2001). La aplicación de las TIC: ¿esnobismo o necesidad educativa?, Red Digital, 1. [en línea http://reddigital.cnice.mecd.es/1/firmas/firmas cabero ind.html]

GONZÁLEZ F. \& SALMON G. (2002). La función y formación del E-moderator: Clave del éxito en los nuevos entornos de aprendizaje. Presentación en Online Educa, Barcelona. [en línea: http://www.atimod.com/presentations/download/educaspanish.doc ]

GRÜNBERG, J. (2002) REDOCENTE: una investigación sobre colaboración electrónica entre docentes de matemáticas y ciencias. Nuevas Tecnologías en Educación, Montevideo, Uruguay Universidad de la República. [en línea: http://www.prc-antel.org.uy/nte/on-line/modulo 3.htm\#3]

Gros, B. (2002). Constructivismo y diseños de entornos virtuales de aprendizaje, Revista de Educación, $328,225-247$.

HARAsim, L., Hiltz, S., TUROFF, M. \&TelES, L. (2000). Redes de aprendizaje: Guía para la enseñanza y el aprendizaje en red, Barcelona:Gedisa/EDIUOC (Versión en Inglés Learning networks. A fiel guide to teaching and learning online. Cambridge (EE.UU.): Massachusetts Institute of Technology,1995)

HARTON, L. \& INGRAM, A. (2002). Cooperation and collaboration using computer mediated communication, Journal Educational Computing Research, 26 (3), 325-347.

HEPP, P.(2003). Enlaces: El programa de informática educativa de la reforma educacional chilena. En COX, C. (Editor) Políticas educacionales en el cambio de siglo: La reforma del sistema escolar de Chile, Santiago, Editorial universitaria, 419-451

KIM, KJ.; BONK, C. J. (2002) Cross-cultural comparisons of online collaboration, Journal of ComputerMediated Communication, 8 (1) [en línea: http://www. ascusc.org/jcmc/vol8/issue1/kimandbonk.html] 
MARCELO, C (2002). La formación inicial y permanente de los educadores. En Consejo Escolar del Estado. Los educadores en la sociedad del siglo XXI, Madrid, Ministerio de Educación, Cultura y Deporte, 161-194.[en línea: http://prometeo.us.es/idea/mie/pub/marcelo/]

MACDONALD, J. (2003). Assessing online collaborative learning: process and product. Computer \& Edutacion, 40 (4), 377-391.

MOORE, M. (Junio 2001). La educación a distancia en los Estados Unidos: estado de la cuestión, ciclo de conferencias sobre el uso educativo de las Tecnologías de la Información y Comunicación y la educación virtual, Universidad Abierta de Cataluña, Barcelona, España.[en línea http://www.uoc.es/web/esp/art/uoc/moore/moore.html ]

PAPERT, S (1996). The Connected family. Bridging the digital generation gap. Atlanta: Longstreet Press.

PÉREZ, A. (2002). Elementos para el análisis de la interacción educativa en los nuevos entornos de aprendizaje, pixel-bit revista de medios y educación, 19, 49-61. [en línea

http://www.sav.us.es/pixelbit/articulos/n19/n19art/art1904.htm]

RyAN, S., ScotT, B., FReEmAN H. \& PATEL, D. (2000). The virtual university: the Internet and resourscebased learning, London: Kogan Page.

SALMON, G. (2000). E-moderating: The key to teaching and learning online, London: Kogan Page.

SALOMON, G \& GLOBERSON, T (1992). Co-participando en el conocimiento: la ampliación de la inteligencia humana con las tecnologías inteligentes. Comunicación, Lenguaje y Educación(13),6-22.

SILVA, J. y OTEIZA, F. (Agosto 2002). Curso a Distancia "Funciones matemáticas en la enseñanza media": Diseño, implementación y los primeros resultados, Actas VI Congreso de Educación a Distancia MERCOSUR/SUL tendencias de la educación a distancia en medio de la revolución informática, Antogafasta, Chile.

Swan, K., Shea, P. Fredericksen, E., Pickett, A. PelZ, W., Maher, G. (2000). Building knowledge building communities: consistency, contact and communication in virtual classroom, Journal Educational Computing Research, 23 (4), 359-381.

VIGOSTKY, L.S.(1978). El desarrollo de los procesos psicológicos superiores, Barcelona:Crítica.

TOLMIE, A. \& Boyle, J. (2000). Factors influencing the success of computer mediated communication (CMC) environments in university teaching: a review and case study, Computers y Education, 34 (2), 119140.

WENGER, E. (2001). Comunidades de práctica. Aprendizaje, significado e identidad. Barcelona: Paidós. 


\title{
Contactar
}

Revista lberoamericana de Educación

\author{
Principal OEI
}

